\title{
Usando regressão logística para estimar o índice de refugo em função de práticas enxutas relacionadas à qualidade
}

\section{Using logistic regression to estimate scrap index in function of quality-related lean practices}

\author{
Graziela dos Santos Bento Mestra em Administração. Universidade Regional de Blumenau (FURB) - Brasil. \\ gsbent080@gmail.com \\ Nelson Hein Doutor em Engenharia de Produção. Universidade Regional de Blumenau (FURB) - Brasil. \\ hein@furb.br.
}

\section{RESUMO}

Este estudo tem como objetivo identificar quais práticas enxutas relacionadas à qualidade possuem maior probabilidade de reduzir o índice de refugo a um valor menor que $5 \%$. A coleta de dados foi realizada por meio de uma survey realizada com profissionais que coordenam áreas de produção ou responsáveis pelos programas de qualidade de 92 indústrias de transformação do Estado de Santa Catarina, no Brasil. Para análise das práticas que mais afetam o índice de refugo da empresa, utilizou-se estatística descritiva e a técnica de regressão logística. Como resultado, identificou-se que níveis mais maduros de utilização de dispositivos à prova de erro nos processos fabris (poka-yokes) e de padronização das atividades aumentam a probabilidade de o índice de refugo ficar abaixo de $5 \%$. As práticas mais maduras são a padronização e a inspeção. Ainda, a maioria das empresas pesquisadas se encontra em um estágio inicial de adoção de práticas enxutas relacionadas à qualidade. Esta pesquisa traz um novo olhar para que gestores possam priorizar as práticas de qualidade com maior efeito na redução do refugo. Embora limitado a um único Estado Brasileiro, o estudo apresenta resultados que podem apoiar os gerentes industriais na escolha de práticas enxutas que melhoram o índice de refugo e, consequentemente, no aumento da qualidade e redução de custos de falhas.

Palavras-chave: Garantia da qualidade. Custos da qualidade. Regressão logística. Refugo. Práticas enxutas.

\section{ABSTRACT}

This study aims to identify which quality-related lean practices are more likely to reduce the scrap index to less than $5 \%$. A survey was applied to collect data from professionals who coordinate production areas or those responsible for quality programs of 92 manufacturing industries in the State of Santa Catarina, in Brazil. Descriptive statistics and the logistic regression technique were used to analyze the practices that most affect the company's scrap index. As a result, it was identified that more mature levels of use of error-proof devices in the manufacturing processes (poka-yokes) and standardization of activities increase the likelihood of the scrap index being below 5\%. The most mature practices are standardization and inspection. Still, most of the companies surveyed are at an early stage of adopting lean practices related to quality. This research brings a new look on the subject so that managers can prioritize quality practices with the most significant effect in reducing waste. Although limited to a single Brazilian state, the study shows results that can help industrial managers choose lean practices that improve the scrap rate and, consequently, increase quality and reduce failure costs.

Keywords: Quality assurance. Cost of Quality. Logistic regression. Scrap. Lean practices. 


\section{INTRODUÇÃO}

A manufatura enxuta tem recebido muito interesse de pesquisa nos últimos anos. A aplicação de práticas enxutas compreende o princípio da redução de custos por meio da eliminação de quaisquer despesas desnecessárias (UHRIN; BRUQUE-CÁMARA; MOYANO-FUENTES, 2017). Por sua vez, produtos de qualidade, com redução de estoque no processo, menos produtos defeituosos e sucata reduzida ajudam a alcançar o desempenho operacional no nível da fábrica. Os indicadores mais comumente utilizados para a medição do desempenho operacional incluem custos, qualidade, entrega e flexibilidade (AHMAD; SCHROEDER, 2003). Ferdows e De Meyer (1990) sugerem que para evitar o dilema de ter que escolher entre um objetivo e outro na produção, é possível construir uma capacidade acumulativa, desde que os recursos sejam primeiramente voltados a melhorar a qualidade. Imai (2014) afirma que a qualidade e custos são compatíveis e que a qualidade é a base sobre o qual o custo e a entrega podem ser desenvolvidos. Assim, a qualidade ainda tem sido reconhecida pelas empresas como um fator competitivo chave para se diferenciar em um mercado globalizado (RAßFELD et al., 2015). Quantificar o custo da qualidade é um dos requisitos para uma implementação completa da Gestão da Qualidade Total (PLEWA; KAISER; HARTMANN, 2016).

Juran (1994) define os custos da qualidade como as falhas na produção que levam a retrabalho, desperdício e perda de produtividade, gerando custos que poderiam ser evitados caso o produto saísse perfeito da primeira vez. De acordo com Plewa, Kaiser e Hartmann (2016), os custos de prevenção abrangem treinamento, certificação de fornecedores, auditoria, modo de falha e análise de efeito e análise de falha de causa raiz. Ainda segundo os autores, os custos de avaliação incluem inspeção de trabalhos em andamento e produtos acabados, técnicos de linha, equipamentos de teste, análise de laboratório e administração relacionada. Finalmente, os custos de falha incluem falha interna - custo de refugo, retrabalho, desperdício etc. - e falha externa - custos de garantia, material devolvido e despesas administrativas.

Nessa perspectiva, a manufatura enxuta é um sistema que pode auxiliar na redução dos custos da qualidade, pois visa eliminar os desperdícios (OHNO, 1997). A qualidade na fonte é um princípio de manufatura enxuta que define que a produção de qualidade não é apenas medida no final da linha de produção, mas em cada etapa do processo produtivo e sendo de responsabilidade de cada indivíduo que contribui para a produção ou entrega de um produto ou serviço no prazo. Significa que a qualidade deve ser incorporada no processo de produção de tal forma que os defeitos sejam identificados e eliminados na sua origem (KUMAR; KUMAR, 2012). Impedir que os produtos defeituosos passem adiante na própria estação de trabalho é mais eficiente do que a inspeção e o retrabalho (LIKER, 2005).

Plewa, Kaiser e Hartmann (2016) identificaram evidências substanciais de que o custo total da qualidade é menor em níveis mais altos de qualidade geral. $\mathrm{O}$ custo da falha diminui em níveis gerais de qualidade mais altos, enquanto a prevenção e os custos de avaliação não são observados como sendo significativamente maiores em níveis gerais de qualidade mais altos.

Nesse contexto, emerge a seguinte pergunta de pesquisa: qual a probabilidade de práticas enxutas relacionadas à qualidade na fonte reduzirem ou ampliarem o índice de refugo? Este estudo tem por objetivo identificar quais práticas enxutas relacionadas à qualidade possuem maior probabilidade de reduzir o índice de refugo a um nível menor que $5 \%$. Portanto, busca-se aprofundar o entendimento de práticas de qualidade relacionadas com a prevenção (garantia da qualidade), avaliação e falha interna (controle da qualidade), contribuindo para tomada de ações gerenciais. Ainda, contribui empiricamente com a temática de custos da qualidade, por meio da análise de indicadores de 92 empresas da indústria de transformação do Estado de Santa Catarina, Brasil. A seguir são apresentadas algumas das práticas mais comumente usadas na indústria manufatureira e associadas ao controle e à garantia da qualidade.

\section{REVISÃO DE LITERATURA}

A qualidade na fonte é a prática da manufatura enxuta preocupada com a redução das perdas relacionadas as medidas de prevenção e controle de defeitos nos produtos. Práticas como a padronização de atividades, a organização da área de trabalho (5S), os dispositivos à prova de erro (poka-yoke), e a rápida 
comunicação de um problema para identificar sua causa, são exemplos de práticas relacionadas à qualidade na fonte.

O trabalho padronizado implica garantir que as instruções de trabalho estejam definidas com detalhes suficientes da operação ou processo e seus parâmetros (KUMAR; KUMAR, 2012). Dennis (2009) cita a estabilidade de processos, a aprendizagem organizacional, o envolvimento dos empregados, a melhoria contínua e o treinamento como benefícios gerados pela aplicação do trabalho padronizado.

Ferramentas para processos e procedimentos padronizados incluem documentos como gráfico de trabalho padronizado, planilha de capacidade de produção, tabela de combinação do trabalho, além de controles visuais, políticas e procedimentos, modelos de amostra, instruções de trabalho, e planilhas de verificação do processo (LIKER; MEIER, 2007). Todas as instruções de trabalho devem conter o movimento padrão do trabalhador, ações, pontos de verificação para qualidade e segurança, junto com o tempo de máquina e inventário padrão (KUMAR; KUMAR, 2012).

Imai (2014) afirma que é impossível melhorar qualquer processo antes que ele seja padronizado. Como parte do processo de solução de problemas, o líder sempre observará o funcionário e verificará a folha de trabalho padronizado. Se o padrão estiver sendo seguido e mesmo assim, defeitos estiverem sendo gerados, há um erro no padrão. A responsabilidade pela melhoria dos padrões deve ser de quem executa o trabalho (LIKER, 2005). O desafio da padronização é encontrar um equilíbrio entre procedimentos rígidos a serem seguidos pelos empregados e liberdade para inovação e criatividade para atingir metas, e isso dependerá da maneira como os padrões são redigidos. Um bom padrão deve ser específico o suficiente para serem guias úteis, mas também gerais o suficiente para permitir alguma flexibilidade (DENNIS, 2009).

Por exemplo, Nissinboim e Naveh (2018) estudaram as condições para que a padronização seja associada à redução de erros. O nível mais alto de redução de erros é encontrado em circunstâncias nas quais os funcionários possuam um grau de flexibilidade para adotarem critérios próprios, a rigidez da padronização é intermediária e, como resultado, a adesão à padronização é alta. Esta situação exige que haja um certo grau de maturidade das práticas de padronização, para permitir a adesão dos empregados. Assim, é possível formular a seguinte hipótese:

H1: Quanto maior a maturidade da padronização, o índice de refugo terá mais chances de apresentar valores inferiores a $5 \%$.

Uma outra forma de padronização que auxilia na prevenção de defeitos é o uso dos $5 \mathrm{~S}$. São cinco etapas: separar, arrumar, limpar, sistematizar e padronizar (IMAI, 2014). O sistema 5 S visa criar um ambiente de trabalho autoexplicativo, que seja fácil de organizar e melhorar (GALSWORTH, 1997). Em um ambiente visual, qualquer situação fora do padrão torna-se imediatamente evidente, tornando a solução de problemas mais fácil (DENNIS, 2009). Dessa forma, ajudam a eliminar as perdas, contribuem para a redução dos erros, defeitos e acidentes de trabalho (LIKER, 2005). Portanto, a segunda hipótese pode ser assim formulada:

H2: Quanto maior a maturidade da aplicação do sistema 5S, o índice de refugo tenderá a valores inferiores a $5 \%$.

Na manufatura enxuta, a definição de cliente inclui tanto os clientes externos, como internos (LIKER, 2005). A filosofia de entender o próximo processo como um cliente, garantindo a qualidade dos produtos em cada etapa da fabricação, é praticada por meio de sistemas à prova de erro - poka-yoke- e da autoridade para parar a linha quando um defeito for identificado (EVERETT; SOHAL, 1991). Os dispositivos à prova de erro, ou poka-yokes, são dispositivos voltados à eliminação dos erros humanos em termos de qualidade, segurança e outros parâmetros do processo para garantir qualidade e segurança nas linhas de fabricação (KUMAR; KUMAR, 2012). As pessoas não cometem erros intencionalmente, mas sim por existir alguma falha no sistema ou método para realizar a tarefa. Isto transfere a culpa pelo erro de operador para o sistema, fazendo com que as pessoas fiquem livres para criarem soluções criativas e eliminarem a causa raíz do problema (LIKER; MEIER, 2007).

Orbak (2012) apresentou um trabalho de redução de refugo no processo de laminação de uma indústria automobilística. Após a introdução de ações de treinamento operacional, dispositivos à prova de erro (poka-yoke) e mudanças no método de movimentação das peças, o refugo foi reduzido em uma média de 3,5 $\%$, com um benefício financeiro de US\$ $45.000,00$ por ano. 
H3: Quanto maior a maturidade da utilização de dispositivos à prova de erro (poka-yokes), o índice de refugo tenderá a valores inferiores a 5\%.

A autoridade do operador para parar a linha de produção é outro aspecto importante. Quando um problema ocorre, o método para resolvê-lo é padronizado. Esse método é chamado de cadeia de ajuda. De acordo com Liker e Meier (2007), o primeiro passo é o reconhecimento de uma situação anormal. O processo de detecção de uma situação fora do padrão é simplificado pelo uso de ferramentas como os padrões visuais, o trabalho padronizado e a autonomação.

A autonomação (jidoka) envolve dar inteligência à máquina, de forma a detectar condições anormais e parar o processo automaticamente, evitando a geração de produtos defeituosos (OHNO, 1997). O empregado tem certa autonomia para resolver o problema, porém quando o tempo de solução ultrapassa o padrão, ele precisa informar o problema e solicitar auxílio da cadeia de ajuda. Para isso, o empregado puxa uma corda, que dispara um andon, dispositivo que possui um alarme sonoro e uma luz para indicar ao líder de equipe a estação onde ocorreu o problema (LIKER; MEIER, 2007).

Este dispositivo permite que o operário pare de produzir imediatamente quando um defeito é detectado, enquanto, ao mesmo tempo, alerta a gerência para a situação (EVERETT; SOHAL, 1991). Neste momento, o líder torna-se responsável pela contenção do problema. Inicia-se então um processo de avaliação do problema para saber sua magnitude e impactos na linha de produção (LIKER; MEIER, 2007).

H4: Quanto maior a maturidade da comunicação dos problemas na linha de produção (andon), o índice de refugo tenderá a valores inferiores a $5 \%$.

O controle da qualidade visa manter o problema na estação e garantir que produtos com defeitos não cheguem ao cliente. Por este motivo, a linha é parada até que o problema seja controlado ou corrigido (OHNO, 1997). A contenção assegura que todas as peças defeituosas sejam corrigidas e auxilia na identificação da fonte do problema. Após o controle e contenção do problema, o foco dirige-se para a prevenção, primeiramente com medidas temporárias, até que medidas permanentes, que eliminem a causa raiz do problema, sejam implementadas e consideradas eficazes (LIKER; MEIER, 2007).

Além disso, a incorporação de inspeções de qualidade em todas as funções por meio da autoinspeção, é baseada em padrões visuais. Isso inclui três atividades básicas: verificar a entrada do trabalho para garantir que não existam defeitos; garantir que defeitos não estão sendo gerados no trabalho atual e nunca passar um produto defeituoso para a operação seguinte conscientemente (LIKER; MEIER, 2007). Assim, atividades de contenção e inspeção, são relacionadas ao custo de avaliação, ou seja, ações necessárias para garantir que os defeitos sejam identificados antes de ir para o cliente.

H5: Quanto maior a maturidade da garantia de que todas as peças, materiais, informações e recursos estejam corretos e atendam às especificações antes de usá-los em um processo, o índice de refugo tenderá a valores inferiores a $5 \%$.

Assim, propõem-se nesse estudo cinco hipóteses a serem testadas, considerando três práticas relacionadas à prevenção (padronização, 5S, poka-yoke) e duas práticas relativas ao controle da qualidade.

\section{METODOLOGIA}

Para identificar os benefícios de um modelo de regressão logística ao se analisar as práticas que mais afetam o índice de refugo de uma empresa, realizou-se uma pesquisa descritiva com abordagem quantitativa por meio de uma survey aplicada em 92 indústrias de manufatura do Estado de Santa Catarina. O Estado de Santa Catarina ocupa posição de destaque no Brasil por meio de um importante parque industrial, representando 28,7\% do PIB do Estado em 2015 (PORTAL DA INDÚSTRIA, 2015).

A população desta pesquisa envolveu organizaçães pertencentes a qualquer ramo de atuação da indústria de transformação catarinense, pertencentes às divisões 10 a 33 da seção $C$ definidas pela Comissão Nacional de Classificação (2016) na CNAE - Classificação Nacional de Atividades Econômicas, como por exemplo papel e celulose, produtos plásticos, metalurgia e produtos de metal, vestuário e alimentos. Foram selecionadas, de maneira equilibrada, indústrias acima de 50 empregados, nacionais ou multinacionais, da 
base de dados da FIESC - Federação das Indústrias do Estado de Santa Catarina. Esta lista resultou em uma população de 800 empresas.

A amostra é não probabilística, intencional e por conveniência. Não probabilística porque os elementos da amostra foram selecionados a critério dos pesquisadores. Intencional, porque os elementos que formam a amostra relacionam-se intencionalmente de acordo com certas características estabelecidas pelo pesquisador (RICHARDSON; PERES, 1985). Finalmente, a amostra também é considerada uma amostra por conveniência, pelo fato de as empresas selecionadas encontrarem-se no estado de domicílio dos pesquisadores, e os contatos de sua rede envolverem profissionais da região, além de grupos de debate sobre o tema específico (HAIR JR. et al., 2005). Sendo assim, a amostra totalizou 92 respondentes, sendo um representante de cada empresa, de 800 questionários enviados, com taxa de retorno de 11,5\%, valor considerado adequado frente aos percentuais indicados por Synodinos (2003).

Profissionais que coordenam áreas de produção ou responsáveis pelos programas de qualidade da empresa, foram convidados por e-mail a participar da pesquisa. A lista de e-mails foi obtida por meio da relação de empresas da FIESC, e rede de networking dos pesquisadores. Além disso, o formulário foi compartilhado num fórum sobre lean na rede social Linkedin, com mais de 31.000 membros. Também foi divulgado para o mailing de alunos e ex-alunos de MBA em Operações e Logística, Especialização em Operações e Logística, Gestão moderna de compras, Engenharia de Produção, Engenharia de Processos, Engenharia de suprimentos e Lean Manufacturing de uma instituição de ensino superior de Joinville.

O instrumento é composto por seis questões objetivas. Os respondentes avaliaram o nível de maturidade das práticas enxutas de qualidade na fonte, por meio da seguinte escala:

1 - Não implantado ou implantando informalmente: O processo ainda não está implantado ou está sendo implantado informalmente, com resultados instáveis.

2 - Formalmente em implantação: Um processo formal de implantação foi iniciado pela empresa. Existe um cronograma de implantação.

3 - Implantado e documentado, com falhas eventuais: O processo está implantado formalmente (documentado), mas existem algumas falhas na execução.

4 - Implantado e documentado com indicadores sob controle: O processo está completamente implantado em uma área ou mais áreas e com indicadores estabelecidos. Os resultados planejados são alcançados.

5 - Implantado, controlado e melhorando continuamente: O processo está completamente implantado, possui indicadores estabelecidos, tem resultados efetivos e exibe melhoria contínua nos últimos 12 meses.

As práticas investigadas foram: andon, qualidade assegurada, padronização das atividades operacionais, poka-yoke e 5S. Tanto as práticas enxutas quanto os níveis de maturidade foram baseados em Santos Bento e Tontini (2018). As questões do instrumento são apresentadas no quadro 1.

\begin{tabular}{|l|l|}
\hline \multicolumn{2}{|c}{ Quadro 1 - Questões para avaliação do nível de maturidade das práticas enxutas } \\
\hline $\begin{array}{l}\text { Qualidadel } \\
\text { assegurada }\end{array}$ & $\begin{array}{l}\text { Questão } \\
\text { e atendam às especificações antes de usá-los em um processo. }\end{array}$ \\
\hline Poka-yoke & $\begin{array}{l}\text { Prevenção de erros humanos, com a introdução de dispositivos à prova de erros na } \\
\text { linha de produção. }\end{array}$ \\
\hline Padronização & $\begin{array}{l}\text { Padronização das tarefas por meio de instruções de trabalho visuais e elaboradas } \\
\text { pelos próprios operadores }\end{array}$ \\
\hline $5 S$ & $\begin{array}{l}\text { Aplicação do } 5 S \text { para limpar e organizar o ambiente de trabalho e garantir que os } \\
\text { materiais e ferramentas necessários estejam prontamente disponíveis e que os } \\
\text { problemas sejam visíveis. }\end{array}$ \\
\hline Andon & $\begin{array}{l}\text { Comunicação de problemas na linha de produção, por meio de sistemas de alerta } \\
\text { sonoro ou visual, para solicitação de ajuda na resolução do problema. }\end{array}$ \\
\hline
\end{tabular}

Fonte: Adaptado de Santos Bento e Tontini (2018). 
Os respondentes informaram ainda, o índice de refugo em termos percentuais da empresa onde atuam, selecionando entre as alternativas "menos de $5 \%$ " ou "acima de $5 \%$ ".

As análises realizadas neste estudo foram suportadas pela aplicação de técnicas estatísticas realizadas no software SPSS 21.0 - Statistical Package for the Social Sciences (IBM, 2013). A primeira análise compreendeu o uso de estatística descritiva realizada para investigação da maturidade das práticas enxutas nas indústrias catarinenses. Para a construção do modelo de análise, empregou-se a técnica estatística de Regressão Logística. Segundo Fávero et al. (2009, p. 440), "a regressão logística é uma técnica estatística utilizada para descrever o comportamento entre uma variável dependente binária e variáveis independentes métrica e não métricas". O fato da variável dependente ser binária ( 0 ou 1 ) possibilita associações de classificação dos fenômenos, como por exemplo rejeitar (0), e, ou aceitar (1) e, também, interpretações em termos de probabilidade de chance de o fenômeno investigado ocorrer ou não ocorrer (DIAS FILHO; CORRAR, 2007).

De acordo com Field (2009), a equação de regressão logística a partir da qual a probabilidade da variável Y é prevista é dada por:

$$
P(Y)=\frac{1}{1+e^{-\left(b_{0}+b_{1} X_{1}+b_{2} X_{2}+\ldots+b_{n} X_{n}+\varepsilon\right)}}
$$

No modelo de regressão logística proposto, a variável dependente é o índice de refugo das empresas e as variáveis independentes representam o conjunto de práticas enxutas que podem influenciar o índice de refugo. O valor de $\mathrm{P}$ significa a probabilidade das práticas enxutas (PES) da qualidade (andon, qualidade assegurada, padronização, poka-yoke, 5S) reduzirem o índice de refugo, ou seja, de o valor de Y ser 1 . Os coeficientes das variáveis independentes $(b)$ representam as variações em unidades que impactam o valor de $\mathrm{P}$ (logaritmo da razão de chance). Assim, a equação que evidencia as variáveis relacionadas no modelo final é definida como:

$$
P(\text { refugo })=\frac{1}{1+e^{-\left(b_{0}+b_{1} P E_{1}+b_{2} P E_{2}+b_{3} P E_{3}+b_{4} P E_{4}+b_{5} P E_{5}+\varepsilon\right)}}
$$

O método de regressão logística utilizado foi a entrada forçada, em que todas as covariáveis são colocadas no mesmo modelo de regressão em um único bloco e as estimativas dos parâmetros são calculadas para cada bloco. O nível de significância adotado foi de 0,05 ( $p$-value $<0,05$ ).

\section{ANÁLISE E DISCUSSÃO DOS RESULTADOS}

O objetivo desse estudo é identificar quais práticas enxutas relacionadas à qualidade possuem maior probabilidade de reduzir o índice de refugo a um nível menor que 5\%.

Primeiramente, foi realizada uma análise descritiva para a amostra total, onde é possível visualizar o nível de maturidade das práticas nas 92 empresas pesquisadas, conforme Tabela 1.

Tabela 1 - Estatística descritiva - Nível de maturidade de práticas enxutas nas empresas pesquisadas

\begin{tabular}{lrrrrr}
\hline & \multicolumn{2}{c}{ Qualidade } & & & \\
& \multicolumn{1}{c}{ Andon } & assegurada & Padronização & Poka-yoke & 5S \\
\hline Média & 2,58 & 2,91 & 3,38 & 2,61 & 2,58 \\
Mediana & 2,50 & 3,00 & 3,00 & 3,00 & 2,50 \\
Moda & 1 & 3 & 3 & 3 & 1 \\
Desvio-padrão & 1,462 & 1,135 & 1,212 & 1,195 & 1,462 \\
\hline
\end{tabular}

Fonte: Dados da pesquisa (2020).

A moda, que é o escore que ocorre mais frequentemente em um conjunto de dados foi utilizada para analisar o nível de maturidade das práticas enxutas predominante nas empresas pesquisadas. Nos casos em que a moda apresentou mais de um número frequente, o menor foi utilizado. 
Considerando-se todas as indústrias da amostra, pode-se verificar na Tabela 1 que as práticas mais incipientes são o andon e os 5S, com a moda no nível 1 de maturidade. São práticas que estão implantadas apenas informalmente, com aplicação em poucas situações. Meiling, Backlund e Johnsson (2012) argumentam que empresas com processos recentes de implantação da manufatura enxuta podem considerar a parada para resolver problemas, como a adoção do andon, uma medida muito drástica, por considerarem apenas a perda de produtividade momentânea em detrimento dos ganhos com a solução dos problemas no longo prazo.

Padronização, qualidade assegurada e o uso de dispositivos à prova de erro (poka-yoke) apresentaram moda 3, ou seja, as práticas foram implementadas, porém os resultados ainda são instáveis. A prática mais madura é a padronização, cuja média é de 3,38.

O próximo passo foi analisar quais práticas enxutas relacionadas à qualidade possuem maior probabilidade de reduzir o índice de refugo a um nível menor que 5\% por meio da técnica de regressão logística, conforme tabela 2. Na primeira rodada, o valor inicial do logaritmo da verossimilhança é de 123,156. A redução desse valor para 98,934 no modelo completo, que inclui as variáveis independentes, indica que o modelo melhorou sua capacidade de predição. Para determinar a qualidade do ajuste que a regressão logística possui usa-se algum pseudo-coeficiente de determinação, como por exemplo o de Nagelkerke ou Cox-Snell, denominados de pseudo- $R^{2}$. As medidas de Cox e Snell, e de Nagelkerke são similares ao $R^{2}$ da regressão (FÁVERO et al., 2009). O $R^{2}$ é considerado fraco entre 0,19 e 0,33; médio entre 0,34 e 0,66; Forte quando maior de 0,67 (HENSELER; RINGLE; SINKOVICS, 2009). Dessa forma, na primeira rodada o $p s e u d o-R^{2}$ de Nagelkerke $(0,314)$ proporciona fraco poder de explicação ao modelo.

Após essa constatação, foi avaliada a estatística de resíduos. Cinco casos da amostra de 92 empresas apresentaram resíduos padronizados próximos a 3,00. Segundo Field (2009), resíduos padronizados acima de 3,00 são motivos de alerta e casos próximos de 3,00 merecem investigação. Dessa forma, os casos sugeridos pelo SPSS foram eliminados da amostra, e uma segunda rodada de análise foi realizada com os 87 casos restantes.

Os resultados da regressão logística melhoraram consideravelmente, mas um caso com resíduo padronizado acima de 3 ainda ocorreu. Após sua exclusão, o modelo acusou apenas dois resíduos com valores padronizados muito próximos de 2,5, ou seja, sem motivos para preocupações. Além disso, os resultados melhoraram consideravelmente em relação ao modelo inicial com 92 casos.

Tabela 2 - Resultados da regressão logística

\begin{tabular}{cccc}
\hline & $\begin{array}{c}\text { Rodada 1 } \\
92 \text { casos }\end{array}$ & $\begin{array}{c}\text { Rodada 2 } \\
87 \text { casos }\end{array}$ & $\begin{array}{c}\text { Rodada 3 } \\
86 \text { casos }\end{array}$ \\
\hline $\begin{array}{c}\text { Verossimilhança de log-2 } \\
\text { Modelo nulo }\end{array}$ & 123,156 & 115,488 & 113,530 \\
\hline $\begin{array}{c}\text { Verossimilhança de log-2 } \\
\text { Modelo completo }\end{array}$ & 98,934 & 68,617 & 62,479 \\
\hline Qui-quadrado & 24,222 & 46,871 & 51,051 \\
\hline Grau de liberdade & 5 & 5 & 5,000 \\
\hline sig & 0,000 & 0,000 & 0,611 \\
\hline Pseudo R ${ }^{2}$ de Nagelkerke & 0,314 & 0,567 & 0,448 \\
\hline Pseudo R ${ }^{2}$ de Cox e Snell & 0,231 & 0,417 & 81,4 \\
\hline $\begin{array}{c}\text { Eficácia classificação } \\
\text { (porcentagem global) }\end{array}$ & 76,1 & 80,5 & 0 \\
\hline$N^{\circ}$ de casos com resíduos acima de 2,6 & 5 & 1 & \\
\hline
\end{tabular}

Fonte: Dados da pesquisa - Saídas SPSS (2020).

Os resultados da rodada 3, apontam ainda que o modelo classifica corretamente $85,2 \%$ dos casos de refugo até $5 \%$, e $75 \%$ dos casos com mais de $5 \%$ de refugo (Tabela 3 ). A acurácia global da classificação é a média ponderada desses dois valores $(81,4 \%)$, o que mostra que o modelo tem uma boa taxa de acertos. 
Tabela 3 - Eficácia de Classificação

\begin{tabular}{|c|c|c|c|c|c|}
\hline & & & & Previsto & \\
\hline & & & & & \\
\hline & Observa & & Até $5 \%$ & Mais de $5 \%$ & Porcentagem correta \\
\hline Etapa 1 & Refugo & Até $5 \%$ & 4 & 8 & 85,2 \\
\hline & & Mais de $5 \%$ & & 24 & 75,0 \\
\hline & Porcent & m global & & & 81,4 \\
\hline
\end{tabular}

Fonte: Dados da pesquisa - Saídas SPSS (2020).

Finalmente, o pseudo- $R^{2}$ de Nagelkerke mostra que as variáveis independentes influenciam $61,1 \%$ nas variações do logaritmo da razão de chance, passando de 0,314 na primeira rodada para 0,611 na terceira.

A tabela 4 apresenta a contribuição individual dos previsores ao modelo. As estatísticas de Wald informam se o coeficiente $B$ de cada previsor é diferente de zero. Se isto ocorrer significa que o previsor está contribuindo significativamente para a previsão da variável dependente (refugo). Ainda, os valores de exp (B) é um indicador da mudança nas probabilidades resultante da mudança de uma unidade no previsor. Se o valor é maior que 1 significa que quando o previsor aumenta, as chances de a saída ocorrer aumentam. Já um valor menor que 1, indica que quando o previsor aumenta, as chances de a saída ocorrer diminuem (FIELD, 2009).

Tabela 4 - Variáveis inseridas na equação

\begin{tabular}{llrrrrrr}
\hline & & B & S.E. & Wald & df & p-value & $\operatorname{Exp(B)}$ \\
\hline \multirow{2}{*}{ Etapa 1a } & PE1 Andon & $-1,680$ & 0,473 & 12,606 & 1 & 0,000 & 0,186 \\
\cline { 2 - 8 } & PE2 Qualidade assegurada & $-2,377$ & 0,677 & 12,306 & 1 & 0,000 & 0,093 \\
\cline { 2 - 8 } & PE3 Padronização & 1,370 & 0,540 & 6,438 & 1 & 0,011 & 3,934 \\
\cline { 2 - 8 } & PE4 Poka-yoke & 1,514 & 0,557 & 7,398 & 1 & 0,007 & 4,545 \\
\cline { 2 - 8 } & PE5 5S & $-0,443$ & 0,333 & 1,771 & 1 & 0,183 & 0,642 \\
\cline { 2 - 8 } & Constante & 2,952 & 1,108 & 7,098 & 1 & 0,008 & 19,147 \\
\hline
\end{tabular}

Fonte: Dados da pesquisa - Saídas SPSS (2020).

O modelo final é expresso pela seguinte equação:

$$
P(\text { refugo })=\frac{1}{1+e^{-(2,952-1,680 \text { andon-2,377QualAsseg }+1,370 \text { padron }+1,514 \text { pokayoke }-0,443 \text { CincoS }+\varepsilon)}}
$$

Com exceção da prática $5 \mathrm{~S}$, todos os demais previsores apresentam a estatística de Wald significativa ( $p$-value<0,05). Os valores de $\operatorname{Exp}(B)$ acima de 1 das variáveis padronização e poka-yoke demonstram que quanto maior a maturidade dessas práticas, maiores as chances de o índice de refugo ficar abaixo dos $5 \%$. Por outro lado, práticas maduras de andon e qualidade assegurada reduzem essa chance.

No caso do Andon, o fato do operador ter autonomia para parar uma linha de produção, evita a proliferação de defeitos e gera uma cultura de melhoria contínua para identificar suas causas. No entanto, é uma medida de controle e contenção, gerando custos de avaliação. Assim, faz-se necessária a correta identificação das causas raízes dos defeitos e tomada de ações para garantir a qualidade na fonte, assegurando que defeitos não serão gerados. Dessa forma, a Hipótese 4 foi rejeitada.

H4: Quanto maior a maturidade da comunicação dos problemas na linha de produção Rejeitada (andon), o índice de refugo tenderá a valores inferiores a 5\%.

Da mesma forma, a qualidade assegurada é uma prática usada para garantir que todas as peças, materiais, informações e recursos estejam corretos e atendam às especificações antes de usá-los em um processo (LIKER; MEIER, 2007). Na maioria das vezes, essa garantia é feita por inspeção de recebimento de matéria-prima ou inspeções na saída dos processos. Portanto, trata-se de uma ação de controle e contenção. Quanto mais inspeções forem realizadas no recebimento e no início de cada processo, o índice de refugo tende a aumentar. Dessa forma, a H5 é rejeitada. 
H5: Quanto maior a maturidade da garantia de que todas as peças, materiais, Rejeitada informações e recursos estejam corretos e atendam às especificações antes de usá-los em um processo, o índice de refugo tenderá a valores inferiores a $5 \%$.

Por outro lado, processos mais maduros de padronização das atividades, com maior participação na elaboração por parte dos empregados, e, consequentemente maior adesão, aumentam a probabilidade do índice de refugo ficar abaixo de 5\%. A hipótese 1 é aceita, e o resultado confirma a ideia de Nissinboim e Naveh (2018) de que níveis mais altos de redução de erros é encontrado em circunstâncias nas quais a adesão dos empregados aos padrões é alta.

H1: Quanto maior a maturidade da padronização, o índice de refugo terá mais chances de apresentar valores inferiores a 5\%.

A introdução de dispositivos à prova de erro nos processos fabris (poka-yokes) também apresenta maiores chances de o índice de refugo ficar abaixo de 5\%. Esta hipótese $(\mathrm{H} 3)$ foi aceita, em linha com a teoria e com achados de estudos anteriores, como os de Orbak (2012).

H3: Quanto maior a maturidade da utilização de dispositivos à prova de erro (poka- $\quad$ Aceita yokes), o índice de refugo tenderá a valores inferiores a $5 \%$.

Finalmente, a prática de $5 \mathrm{~S}$ não prevê de forma significativa o refugo na amostra pesquisada. Apesar dos benefícios comprovados em se manter o ambiente de trabalho limpo e organizado (LIKER, 2005), não houve associação de práticas de $5 \mathrm{~S}$ com o índice de refugo abaixo de 5\%. Portanto, rejeita-se a hipótese $\mathrm{H} 2$.

H2: Quanto maior a maturidade da aplicação do sistema 5S, o índice de refugo tenderá Rejeitada a valores inferiores a $5 \%$.

\section{CONCLUSÕES}

Esta pesquisa teve como objetivo entender quais práticas enxutas relacionadas à qualidade na fonte possuem maior probabilidade de reduzir o índice de refugo a um nível menor que $5 \%$. Um dos principais achados da pesquisa pode ser considerado o fato de nem todas as práticas enxutas relacionadas à qualidade aumentam as chances de redução do refugo a níveis inferiores a 5\%. Enquanto as práticas relacionadas à prevenção como padronização de tarefas e adoção de dispositivos à prova de erros aumentam essa chance, práticas de controle da qualidade como a comunicação de problemas na linha de produção para resolução rápida de problemas, e a garantia de que todas as peças, materiais, informações e recursos estejam corretos antes de usá-los em um processo, reduzem as chances de baixos índices de refugo.

Outro achado importante é que a maioria das empresas catarinenses se encontra em um estágio inicial de adoção de práticas enxutas relacionadas à qualidade na fonte, no qual a ocorrência de falhas é frequente e os resultados são instáveis. As práticas mais maduras são a padronização e a qualidade assegurada. Já as práticas menos maduras surpreendentemente estão relacionadas ao estabelecimento de métodos e participação de pessoas, não havendo necessidade de altos investimentos para alcançar resultados rápidos, como por exemplo, os 5S, a aplicação de dispositivos à prova de erros na linha de produção (poka-yokes), e a comunicação de problemas na linha de produção, por meio de sistemas de alerta sonoro ou visual (andon).

Destaca-se como contribuição o uso da abordagem quantitativa para estimar o índice de refugo em função de práticas de prevenção e avaliação da qualidade indústrias de transformação do Estado de Santa Catarina, uma vez que a maioria das pesquisas com indústria nesta região são estudos de caso ou consideram apenas um segmento. Outra contribuição é a metodológica, uma vez que não foram identificados na revisão 
da literatura outros estudos que usassem a regressão logística na investigação de práticas relacionadas à qualidade e indicadores de desempenho.

Como implicações gerenciais, esta pesquisa traz um novo olhar para que gestores possam priorizar as práticas de qualidade preventivas, com maior efeito na redução do refugo. Serve também como um alerta de que práticas de controle de qualidade são importantes para evitar que produtos com problema sigam para o cliente. No entanto, aumentam a probabilidade das taxas maiores de refugo.

Um dos principais desafios encontrados para realização deste estudo foi a dificuldade de conseguir resposta das empresas, fato que pode ser proveniente do desejo de manterem sigilo em relação ao seu desempenho. Apesar do esforço para preservar a confidencialidade e privacidade dos respondentes, alguns contatos alegaram não poder participar devido às políticas estabelecidas pela empresa. Além disso, existe a possibilidade de viés nas respostas devido ao fato de apenas um representante de cada empresa ter respondido a pesquisa.

A validade dos achados poderia ser melhorada no futuro estendendo a amostra para outros estados brasileiros. Além disso, outras práticas da manufatura enxuta podem ser correlacionadas com diferentes indicadores de desempenho operacional, como custo, confiabilidade e flexibilidade, para a construção de um mapa de priorização de implementação de ferramentas enxutas, de acordo com o resultado esperado pela empresa.

\section{REFERÊNCIAS}

AHMAD, Sohel; SCHROEDER, Roger G. The impact of human resource management practices on operational performance: recognizing country and industry differences. Journal of operations Management, v. 21, n. 1 , p. 19-43, 2003.

DENNIS, Pascal. Produção lean simplificada. Porto Alegre: Bookman Editora, 2009.

DIAS FILHO, J. M.; CORRAR, L. J. Regressão logística. In: CORRAR, L. J; PAULO, E.; DIAS FILHO, J. M. (org.). Análise multivariada: para os cursos de administração, ciências contábeis e economia. São Paulo: Atlas, 2007. p. 280-323.

SANTOS BENTO, Graziela dos; TONTINI, Gérson. Developing an instrument to measure lean manufacturing maturity and its relationship with operational performance. Total Quality Management \& Business Excellence, p. 1-19, 2018.

EVERETT, R. J.; SOHAL, A. S. Individual involvelment and intervention in Quality Improvement Programmes: Using the Andon System. International Journal of Quality \& Reliability Management, v. 8, n. 2, p. 21-34, 1991.

FÁVERO, L. P. et al. Análise de dados: modelagem multivariada para tomada de decisões. Rio de Janeiro: Elsevier, 2009.

FERDOWS, Kasra; DE MEYER, Arnoud. Lasting improvements in manufacturing performance: in search of a new theory. Journal of Operations Management, v. 9, n. 2, p. 168-184, 1990.

FIELD, Andy. Descobrindo a estatística usando o SPSS. 2. ed. Porto Alegre: Artmed, 2009.

GALSWORTH, Gwendolyn D. Visual systems: harnessing the power of the visual workplace. New York: American Management Association, 1997.

HAIR JR, J. F. et al. Fundamentos de métodos de pesquisa em administração. Porto Alegre: Bookman, 2005. 
HENSELER, Jörg; RINGLE, Christian M.; SINKOVICS, Rudolf R. The use of partial least squares path modeling in international marketing. In: CAVUSGIL, Tamer; SINKOVICS, Rudolf R.; GHAURI, Pervez N. (org.). New challenges to international marketing. Bingley: Emerald Group Publishing Limited, 2009. p. 277-320.

IBM Corp. IBM SPSS Statistics for Windows: Version 22.0. Armonk, NY: IBM Corp, 2013.

IMAI, Masaaki. Gemba Kaizen: uma abordagem de bom senso à estratégia de melhoria contínua. Porto Alegre: Bookman Editora, 2014.

JURAN, Joseph M. A qualidade desde o projeto: novos passos para o planejamento da qualidade em produtos e serviços. 2. ed. São Paulo: Pioneira, 1994.

KUMAR, Rakesh; KUMAR, Vikas. Lean manufacturing: elements and its benefits for manufacturing industry. In: NATIONAL CONFERENCE ON TRENDS AND ADVANCES IN MECHANICAL ENGINEERING, 16., 2012, Faridabad Proceedings [...]. Faridabad: YMCA, 2012. p. 748-755.

LIKER, Jeffrey K. O modelo Toyota:14 princípios de gestão do maior fabricante do mundo. Porto Alegre: Bookman, 2005.

LIKER, Jeffrey K; MEIER, David. O Modelo Toyota-Manual de Aplicação: um guia prático para a implementação dos 4Ps da Toyota. Porto Alegre: Bookman Editora, 2007.

MEILING, John; BACKLUND, Fredrik; JOHNSSON, Helena. Managing for continuous improvement in off-site construction: Evaluation of lean management principles. Engineering,

Construction and Architectural Management, v. 19, n. 2, p. 141-158, 2012.

NISSINBOIM, Noa; NAVEH, Eitan. Process standardization and error reduction : A revisit from a choice approach. Safety Science, v. 103, p. 43-50, mar. 2018.

OHNO, Taiichi. O sistema Toyota de produção além da produção. Porto Alegre: Bookman, 1997.

ORBAK, Ali Y. Shell scrap reduction of foam production and lamination process in automotive industry. Total Quality Management \& Business Excellence, v. 23, n. 3, p. 325-341, 2012.

PLEWA, Markus; KAISER, Gernot; HARTMANN, Evi. Is quality still free? Empirical evidence on quality cost in modern manufacturing. International Journal of Quality \& Reliability Management, v. 33, n. 9, p. 1270$1285,2016$.

PORTAL DA INDÚSTRIA. Participação industrial: Santa Catarina, 2015. 2015. Disponível em: http://perfildaindustria.portaldaindustria.com.br/estado/sc. Acesso em: 5 out. 2018.

RAßFELD, C. et al. Do quality costs still matter? Total Quality Management \& Business Excellence, v. 26, n. 9-10, p. 1071-1082, 2015.

RICHARDSON, Roberto Jarry; PERES, José Augusto. Pesquisa social: métodos e técnicas. São Paulo: Atlas, 1985.

SYNODINOS, Nicolaos E. The "art" of questionnaire construction: some important considerations for manufacturing studies. Integrated Manufacturing Systems, v. 14, n. 3, p. 221-237, 2003.

UHRIN, Ákos; BRUQUE-CÁMARA, Sebastian; MOYANO-FUENTES, José. Lean production, workforce development and operational performance, Management Decision, v. 55 n. 1, p.103-118, 2017. 